\title{
Development of a synthetic phantom for the selection of optimal scanning parameters in CAD-CT colonography
}

\author{
Tarik A. Chowdhury ${ }^{\mathrm{a}, *}$, Paul F. Whelan ${ }^{\mathrm{a}}$, Ovidiu Ghita ${ }^{\mathrm{a}}$, \\ Nicolas Sezille ${ }^{\mathrm{a}}$, and Shane Foley ${ }^{\mathrm{b}}$ \\ ${ }^{a}$ Vision Systems Group, Dublin City University, Dublin 9, Ireland \\ ${ }^{\mathrm{b}}$ Department of Radiology, Mater Misericordiae Hospital, Dublin 7, Ireland
}

\begin{abstract}
The aim of this paper is to present the development of a synthetic phantom that can be used for the selection of optimal scanning parameters in Computed Tomography (CT) colonography. In this paper we attempt to evaluate the influence of the main scanning parameters including slice thickness, reconstruction interval, field of view, table speed and radiation dose on the overall performance of a Computer Aided Detection (CAD)-CTC system. From these parameters the radiation dose received a special attention, as the major problem associated with $C T C$ is the patient exposure to significant levels of ionising radiation. To examine the influence of the scanning parameters we performed $51 \mathrm{CT}$ scans where the spread of scanning parameters was divided into seven different protocols. A large number of experimental tests were performed and the results analysed. The results show that automatic polyp detection is feasible even in cases when the $C A D-C T C$ system was applied to low dose CT data acquired with the following protocol: $13 \mathrm{mAs} /$ rotation with collimation of $1.5 \times 16 \mathrm{~mm}$, slice thickness of $3.0 \mathrm{~mm}$, reconstruction interval of $1.5 \mathrm{~mm}$, table speed
\end{abstract}


of $30 \mathrm{~mm}$ per rotation. The CT phantom data acquired using this protocol was analysed by an automated CAD-CTC system and the experimental results indicate that our system identified all clinically significant polyps (i.e. larger than $5 \mathrm{~mm}$ ).

Key words: CT Colonography, synthetic phantom, radiation dose, polyp detection.

\section{Introduction}

Colon cancer is the second leading cause of cancer deaths in the developed nations [1-3] and numerous studies indicated that early detection and removal of colon polyps is the most effective way to reduce colorectal cancer (CRC) mortality [4-7]. Colonoscopy is widely considered the standard diagnostic technique for the detection of colonic neoplasia $[8,9]$ but it is important to mention that colonoscopy is a highly invasive and time consuming medical investigation [10]. Virtual colonoscopy (VC) or CT colonography (CTC) [1114] is a minimally invasive medical procedure that has been proposed as an alternative to conventional colonography. Since its introduction by Vining et al. [11] in 1994, CTC has received extensive attention from research community and many publications have emerged in areas of 3D surface rendering and visualization [15-17], centerline calculation [18], colon unfolding [19] and automated polyp detection [20-34]. Recent publications [22,23,35] indicate that the results returned by the automatic $C A D-C T C$ polyp detection systems in the vast majority of cases closely match or even outperform the human reader performance. It is worth mentioning that the performance of the $C A D-C T C$ systems is constantly improving and this is driven not only by the develop-

* Corresponding author.

Email address: tarik@eeng.dcu.ie (Tarik A. Chowdhury). 
ment of new more sophisticated algorithms for polyp detection but also by the advances in the development of the CT scanners. From this aspect, the current range of the MDCT (Multi-Detector CT) scanners offer excellent image quality and the typical acquisition period is reduced to 20-30 seconds for a full abdominal scan.

The major concern associated with CTC is the fact that the patients are subjected to high levels of ionising radiation. The medical literature indicates that the level of ionising radiation received by the patients during the $\mathrm{CT}$ examination varies from 5 to $20 \mathrm{mSv}$ [36-41] and this radiation level may induce cancer in $0.05 \%$ of the patients older than 50 years that were subjected to a CT abdominal examination [42]. Cohen [43] indicates in his paper that the risk of inducing cancer in patients is significantly lowered when they are subjected to low-level radiation exposure and an important number of studies were carried out in order to identify the minimal level of radiation dose that can be used in CTC but without a negative impact on the detection of colorectal polyps $[41,44-46]$. The identification of the optimal scanning parameters (collimation, slice thickness, table speed, reconstruction interval) is a difficult problem and this procedure is applied on synthetic phantoms that are designed to accurately model the human body [47-58]. In this sense, Beaulieu et al. [47] used spherical plastic beads to model polyps while Dachman et al. [48] created false polyps in a pig colon by puckering the mucosa of the colon. Their studies focused on finding the imaging effect of collimation, tube current (pitch) and orientation when they analysed different sizes and types of polyps. Similar studies were performed by Taylor et al. [49] and Springer et al. [50]. Using a different approach, Whithing et al. [51] constructed an air filled acrylic cylinder where polyps of different sizes were attached on the 
inner side of the acrylic tube and they applied the developed phantom to evaluate the artefacts generated by the collimation and the tube current. Laghi et al. [54] and Embleton et al. [55] used synthetic and pig colons and their tests indicate that CT scans with a collimation of $4 \times 2.5 \mathrm{~mm}, 1.25 \mathrm{~mm}$ reconstruction interval, $40 \mathrm{mAs} /$ rotation generate datasets with sufficient resolution to be used for automated and manual CTC polyp detection. Ozgun et al. [56] used latex material to build phantom polyps having dimensions ranging from $1 \mathrm{~mm}$ to $10 \mathrm{~mm}$. Their tests were focused on finding the minimal tube current that allows the detection of polyps larger than $5 \mathrm{~mm}$. They reported that the detection of the polyps larger than $5 \mathrm{~mm}$ is feasible only if the CT scans are performed in the range 60 to $100 \mathrm{mAs} /$ rotation.

In this paper we evaluate the effect of key scanning parameters (mAs/rotation, slice thickness, reconstruction interval, field of view and table speed) by analysing the CT data obtained by scanning a novel synthetic phantom. The phantom has been specifically designed for $C A D-C T C$ to simulate colon polyps with different shapes (pedunculated, sessile and flat) and sizes (3 to $18 \mathrm{~mm})$. In our studies the CT phantom data is evaluated using an automated $C A D-C T C$ system [34] in order to determine the influence of the scanning parameters on polyp detection. A special emphasis of our study is placed on determining the minimal radiation dose that allows robust identification of colonic polyps but not at the expense of reduced sensitivity in polyp detection. This paper is organised as follows. In Section 2 the development of the synthetic phantom is detailed. Section 3 briefly presents the automated $C A D-C T C$ polyp detection system. In Section 4 the experimental results are presented and discussed while Section 5 concludes this paper. 


\section{Materials and Methods}

\subsection{Phantom design}

A synthetic phantom was constructed using a PVC tube, two acrylic tubes, two plastic plates and latex material to emulate the colon wall, polyps and folds. The external PVC tube is $230 \mathrm{~mm}$ long with a diameter of $300 \mathrm{~mm}$. Acrylic tubes are $235 \mathrm{~mm}$ long and the dimensions of the inner and outer diameters are $40 \mathrm{~mm}$ and $50 \mathrm{~mm}$ respectively. Hounsfield Unit (HU) values of the PVC tube, acrylic tubes and plastic plates are 1500, 100, 90 respectively. The construction of the synthetic phantom is illustrated in Figure 1.

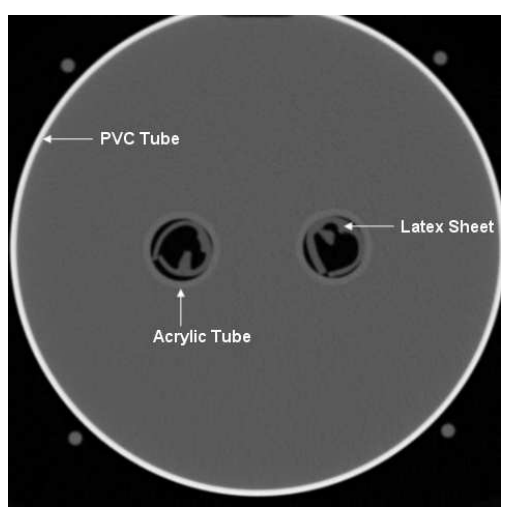

(a)

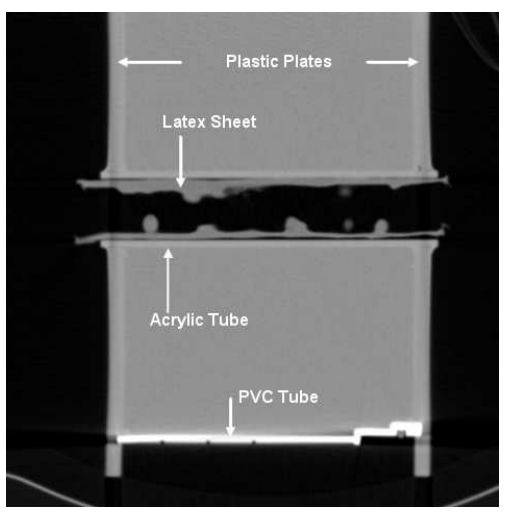

(b)

Fig. 1. Synthetic colon phantom. (a) Longitudinal view. (b) Transversal view.

The polyp inserts for phantom were made using latex material having a $H U$ value of -95 . We have chosen to use latex as this material allows us to generate very realistic shapes (pedunculated, sessile, flat, flat-depressed) for polyps and folds as illustrated in Figure 2. In addition the $H U$ values associated with the latex material approximate the $H U$ values of the colon wall $(\sim 10 \mathrm{HU})$. In CTC 
the large difference between the $H U$ values associated with the air voxels ($1000 \mathrm{HU}$ ) and the $H U$ values of the colon tissue are evaluated to identify the surface of the colon wall. The model for polyps was made from clay and liquid latex was poured into the model to create the latex polyp inserts. To make the surface of the latex sheet more realistic the thickness of the sheet was made uneven. We have created two sheets of latex containing 48 polyps having different sizes, 7 flat polyps, 2 depressed flat polyps, 15 non-spherical polyps, 2 pedunculated polyps, 22 spherical/elliptical polyps and 6 haustral folds. In Figure 3 several 3D views of some representative synthetic polyps are depicted.

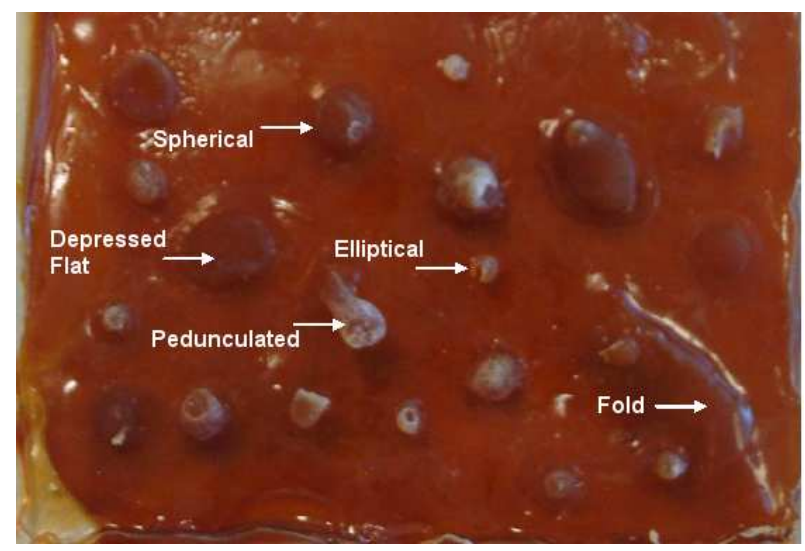

Fig. 2. Latex insert sheet with various types of polyps and folds.

\subsection{Image acquisition}

The developed phantom described in Section 2.1 was scanned using a 16-slice Siemens Somatom Sensation CT scanner in Mater Hospital, Dublin, Ireland. The phantom has been scanned in longitudinal (phantom was placed parallel to the CT scanner bed) and transversal directions, where the scanning 


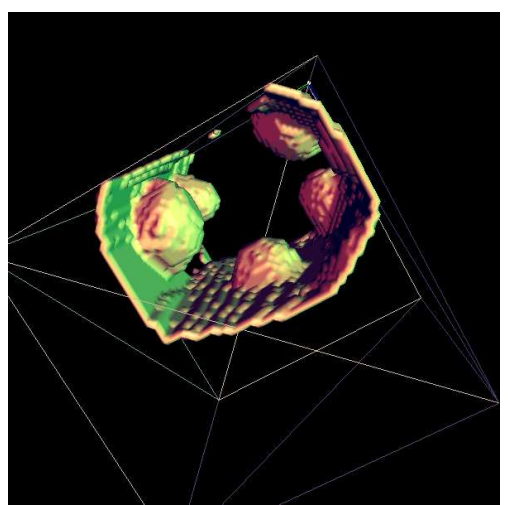

(a)

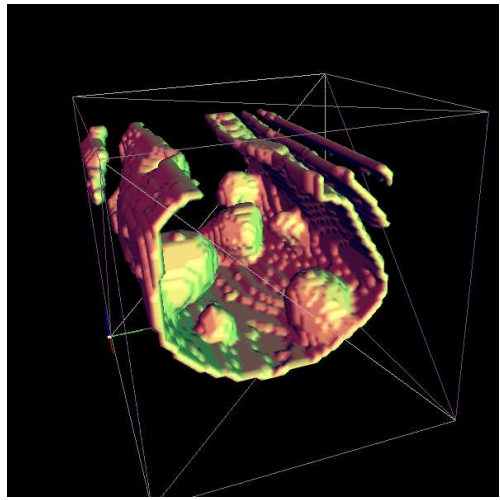

(c)

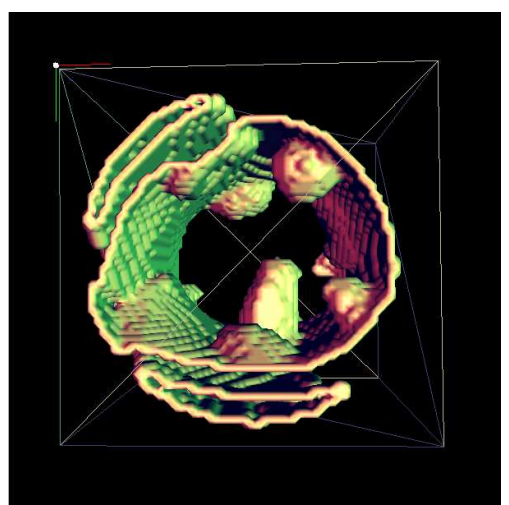

(b)

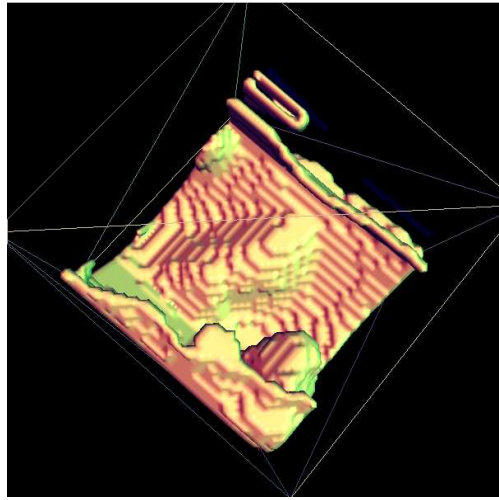

(d)

Fig. 3. 3D longitudinal views of the synthetic polyps (a-c) and fold (d) made from latex.

parameters (collimation, slice thickness, field of view, table speed, reconstruction interval and mAs/rotation) were varied. All scans were performed at $120 \mathrm{kVp}$ tube voltage. It is useful to note that the effective radiation dose is influenced by the value of the tube voltage but its relationship with image quality, tissue contrast and image noise is complex and the effect of this parameter would be difficult to be evaluated. Therefore, in our experiments we maintained the value of this parameter constant $(120 \mathrm{kVp})$ because this is the standard value of the tube voltage used in most clinical examinations. The 
smoothing reconstruction filter used was the B30 filter [59] and this filter has been employed based on its optimal performance in data smoothing and noise removal (this is the filter used in most clinical studies and a detailed evaluation on the performance of the available smoothing filters is beyond the scope of this investigation).

In conjunction with our clinical partners from Mater Hospital we have chosen the following spread of parameters: collimation $0.75 \times 16 \mathrm{~mm}$ and $1.5 \times 16 \mathrm{~mm}$, field of view: 325 and $360 \mathrm{~mm}$, table speed: 20 to $30 \mathrm{~mm} /$ rotation, slice thickness of 1, 2 and 3mm and mAs/rotation: 100, 80, 70, 60, 50, 40, 30, 20 and 13 (13 mAs/rotation is the minimum value that can be set for Siemens Somatom Sensation CT scanner used in our experiments). These scanning parameters have been divided into seven protocols as follows:

- Protocol 1: Collimation $1.5 \times 16 \mathrm{~mm}$, slice thickness $3 \mathrm{~mm}$, reconstruction interval $1.5 \mathrm{~mm}$, field of view $325 \mathrm{~mm}$, table speed $30 \mathrm{~mm} /$ rotation, $\mathrm{mAs} /$ rotation: $100,80,70,60,50,40,30,20$ and 13 . This protocol was used to identify the effect of radiation dose and scan orientation (longitudinal and transversal scans) on the performance of our automatic $C A D-C T C$ system.

- Protocol 2: Collimation $1.5 \times 16 \mathrm{~mm}$, slice thickness $3 \mathrm{~mm}$, reconstruction interval $1.5 \mathrm{~mm}$, field of view $360 \mathrm{~mm}$, table speed $30 \mathrm{~mm} /$ rotation, $\mathrm{mAs} /$ rotation: 50, 30, 20 and 13. This protocol was employed to evaluate the influence of the field of view and the variation of the radiation dose.

- Protocol 3: Collimation $1.5 \times 16 \mathrm{~mm}$, slice thickness $3 \mathrm{~mm}$, reconstruction interval $1 \mathrm{~mm}$, field of view $325 \mathrm{~mm}$, table speed $30 \mathrm{~mm} /$ rotation, $\mathrm{mAs} /$ rotation: $100,80,70,60,50,40,30,20$ and 13 . This protocol was used to analyse the effect of the reconstruction interval and the radiation dose.

- Protocol 4: Collimation $1.5 \times 16 \mathrm{~mm}$, slice thickness $2 \mathrm{~mm}$, reconstruction in- 
terval $1 \mathrm{~mm}$, field of view $325 \mathrm{~mm}$, table speed $30 \mathrm{~mm} /$ rotation, $\mathrm{mAs} /$ rotation: 100, 50, 40, 30, 20 and 13. This protocol was used to generate CT data where the effect of the slice thickness and the radiation dose is analysed.

- Protocol 5: Collimation $1.5 \times 16 \mathrm{~mm}$, slice thickness $2 \mathrm{~mm}$, reconstruction interval $0.8 \mathrm{~mm}$, field of view $325 \mathrm{~mm}$, table speed $30 \mathrm{~mm} /$ rotation, $\mathrm{mAs} /$ rotation: 100, 50, 40, 30, 20 and 13. This protocol was employed to analyse the joint effect of the slice thickness, reconstruction interval and radiation dose.

- Protocol 6: Collimation $1.5 \times 16 \mathrm{~mm}$, slice thickness $3 \mathrm{~mm}$, reconstruction interval $1.5 \mathrm{~mm}$, field of view $325 \mathrm{~mm}$, table speed $20 \mathrm{~mm} /$ rotation, $\mathrm{mAs} /$ rotation: 100, 50, 40, 30 and 20. This protocol was used to find the effect of table speed at different radiation doses.

- Protocol 7: Collimation $0.75 \times 16 \mathrm{~mm}$, slice thickness $1 \mathrm{~mm}$, reconstruction interval $0.7 \mathrm{~mm}$, field of view $325 \mathrm{~mm}$, table speed $30 \mathrm{~mm} /$ rotation, mAs/rotation: 100, 60, 40, 30 and 22. This protocol was used to find the effect of collimation and radiation doses on the performance of our automatic $C A D-C T C$ system.

\section{CAD-CTC polyp detection algorithm}

We have developed an automated $C A D-C T C$ method designed to identify the colorectal polyps in CT data [34] that evaluates the local morphology of the colon wall. Initially, the colon is segmented using a seeded 3D region growing algorithm that was applied to segment the air voxels, which assures the robust identification of the colon wall. In some situations the colon is collapsed due to either insufficient insufflation or residual water. In order to address this issue we have developed a novel colon segmentation algorithm 
that is able to correctly identify the colon segments using knowledge about their sizes and location within the body in all imaging conditions (for more details refer to [60]). After the identification of the colon wall, the normal vector is calculated for each voxel of the colon wall using the Hummel-Zucker operator [61]. The normal vectors sample the local orientation of the colonic surface and the suspicious candidate structures that may resemble polyps are extracted using a simple convexity analysis. The suspicious colonic surfaces (candidate surfaces) have convex properties and are detected using the 3D histogram and the Gaussian distribution of the Hough Points (for a detailed description of this algorithm refer to [34]). This method is able to correctly identify all polyps above $3 \mathrm{~mm}$ but it is worth noting that this is achieved at the cost of a high level of false positives. In order to reduce the level of false positives, statistical features [34] including the standard deviation of surface variation, ellipsoid fitting error, sphere fitting error, three axes of the ellipsoid and the Gaussian sphere radius are calculated for each candidate surface that has been identified by the convexity method described before. These features are fed into a feature normalised classifier [62] that is able to decide whether the surface under investigation belongs to a polyp or a fold. The classifier was trained using a collection of 64 polyps and 354 folds that were selected by a radiologist. The developed $C A D-C T C$ algorithm was tested on phantom (standard and low dose CT datasets) and real patient data (mAs/rotation of 100) and shows $100 \%$ sensitivity for polyps larger than $5 \mathrm{~mm}$ with a rate of 4.05 false positives per dataset. 


\section{Experiments and results}

The aim of this paper is to evaluate the influence of the scanning parameters on the overall polyp detection results in $C A D-C T C$ systems. In order to evaluate this, the synthetic phantom detailed in Section 2 has been scanned and a total of $51 \mathrm{CT}$ datasets have been acquired using the seven protocols mentioned in Section 2.2.

When the $C A D-C T C$ system has been applied to CT data acquired using the Protocol 1, the results indicate that $100 \%$ sensitivity has been achieved for polyps larger than $10 \mathrm{~mm}$ in both longitudinal and transversal positions for all radiation levels (100 to $13 \mathrm{mAs} /$ rotation). For medium size polyps (5mm to $10 \mathrm{~mm}$ ) the sensitivity was $100 \%$ in all cases but 20 and $30 \mathrm{mAs} /$ rotation, where the sensitivity rate was $95 \%$. The reduction in sensitivity was caused by the undetected polyp illustrated in Figure 4a which was situated close to the end plates. The sensitivity in polyp detection when the $C A D-C T C$ algorithm was applied to $C T$ data acquired using the Protocol 1 is illustrated in Figure 5.

The sensitivity of the polyp detection achieved when the $C A D-C T C$ algorithm has been applied to CT data acquired using the Protocol 2 is $100 \%$ for polyps larger than $10 \mathrm{~mm}$. The sensitivity for medium size polyps ( 5 to $10 \mathrm{~mm}$ ) dropped to $95 \%$ when the phantom was scanned with 30, 20 and 13 mAs/rotation. There was only one polyp undetected for data acquired with this protocol and is illustrated in Figure $4 \mathrm{~b}$.

For CT data acquired using the Protocol 3, the polyp detection for all scans show $100 \%$ sensitivity except the case when the phantom has been scanned with $30 \mathrm{mAs} /$ rotation. The polyp undetected is illustrated in Figure 4b. The polyp detection sensitivity when the scans were performed using the Pro- 


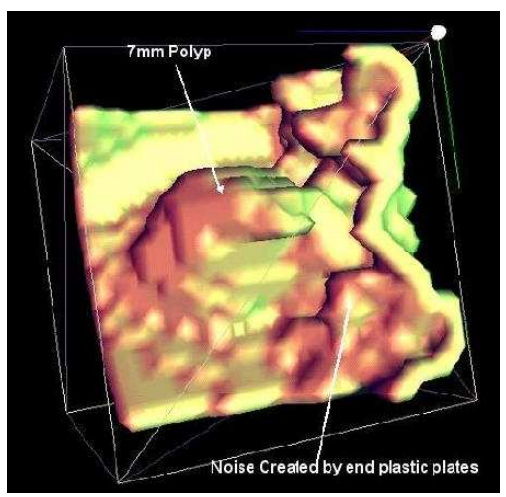

(a)

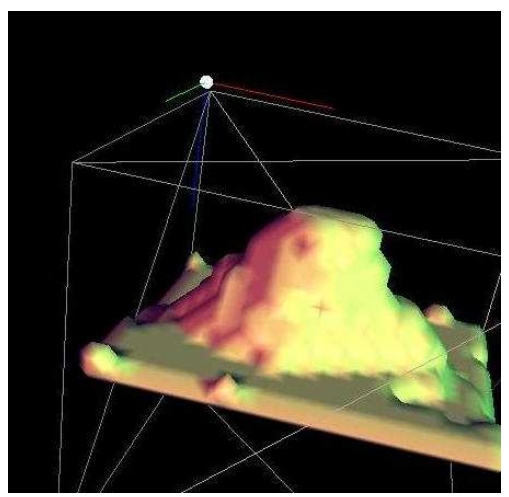

(b)

Fig. 4. (a) Polyp undetected by the $C A D-C T C$ algorithm when the data was acquired using the Protocols 1, 3 and 6. (b) Polyp undetected by the CAD-CTC algorithm when the data was acquired using the Protocols 2, 4 and 5.

tocol 4 is $100 \%$ for polyps larger than $10 \mathrm{~mm}$ for all radiation doses except $100 \mathrm{mAs} /$ rotation. The sensitivity in polyp detection for medium size polyps is also $100 \%$ except the case when the phantom has been scanned with $30 \mathrm{mAs} /$ rotation when the sensitivity dropped to $95 \%$. The polyp missed by the $C A D-C T C$ system is illustrated in Figure 4a. The sensitivity in polyp detection obtained when the $C A D-C T C$ system was applied to CT data scanned using the Protocol 5 is lower than the sensitivity obtained when the Protocols 1 to 4 were employed. The reason for this is that no interpolation was applied to obtain an isometric dataset as the reconstruction interval is $0.8 \mathrm{~mm}$ and the voxel resolution is almost the same in all directions (the lower performance of the $C A D-C T C$ system when applied to datasets acquired using the Protocol 5 is justified since the classifier is trained only with interpolated data). Sensitivity achieved for polyp detection when the $C A D-C T C$ algorithm has been applied to CT data obtained using the Protocol 6 is $100 \%$ for all radiation doses except the case when the data is scanned with $20 \mathrm{mAs} /$ rotation. The polyp missed by 
Sensitivity of Polyp Detection for Protocol-1 Longitudinal CT data

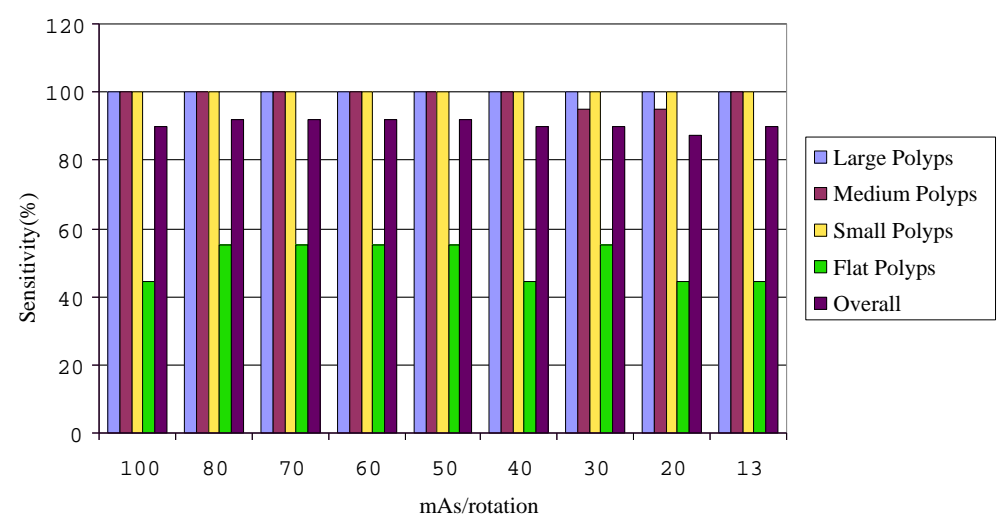

(a)

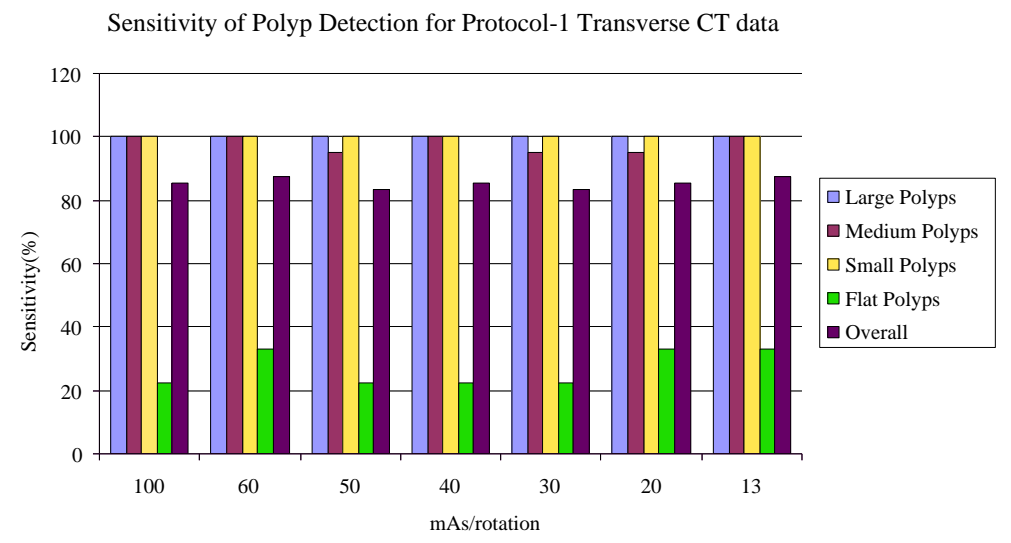

(b)

Fig. 5. Sensitivity of the polyp detection algorithm when applied to CT data (Protocol-1: Collimation $1.5 \times 16 \mathrm{~mm}$, slice thickness $3 \mathrm{~mm}$, reconstruction interval $1.5 \mathrm{~mm}$, field of view $325 \mathrm{~mm}$, table speed $30 \mathrm{~mm} /$ rotation) acquired at different radiation doses. (a) and (b) show the sensitivities for Protocol-1 longitudinal and transversal CT data respectively.

the polyp detection algorithm is illustrated in Figure 4a. For CT data acquired using the Protocol 7, the polyp detection for all scans shows $100 \%$ sensitivity for polyps $\geq 10 \mathrm{~mm},[5-10) \mathrm{mm}$ and $<5 \mathrm{~mm}$. Results of the automated polyp 
detection for all 51 scans used in our experiments are depicted in Figures 5 to 11 . It is useful to note that the overall sensitivity achieved by our $C A D$ CTC system is lowered by the inclusion of flat polyps. The sensitivity rate for flat polyps is between $22 \%$ to $55 \%$ and our method has not been designed to detect this class of colorectal polyps. The flat polyps have distinct shapes and their identification should be approached by a $C A D-C T C$ system that is specifically designed to deal with this type of polyps [63].

Sensitivity of Polyp Detection for Protocol-2 CT data

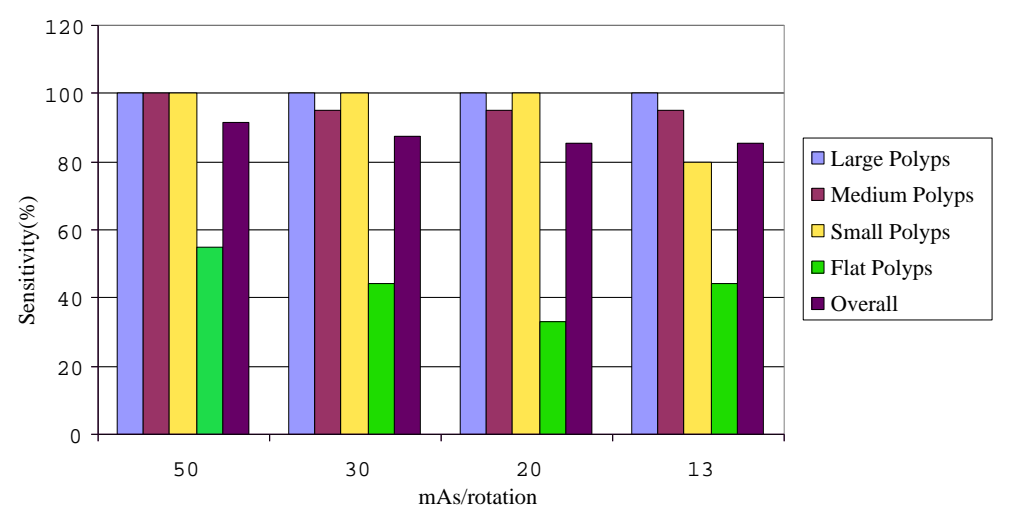

Fig. 6. Sensitivity of the polyp detection algorithm when applied to Protocol-2 CT data.

\subsection{Effect of slice thickness, reconstruction interval and table speed}

To analyse the effect of slice thickness and reconstruction interval, the synthetic phantom has been scanned using protocols where these parameters are varied (Protocols 1,3,4,5 and 7). An important step preceding the application of the $C A D-C T C$ algorithm is data interpolation. All CT datasets were interpolated in order to make them isometric except cases when they were obtained when the phantom was scanned using the Protocol 5. The CT data obtained using the Protocol 5 was not interpolated as the voxel resolution is almost 
Sensitivity of Polyp Detection for Protocol-3 CT data

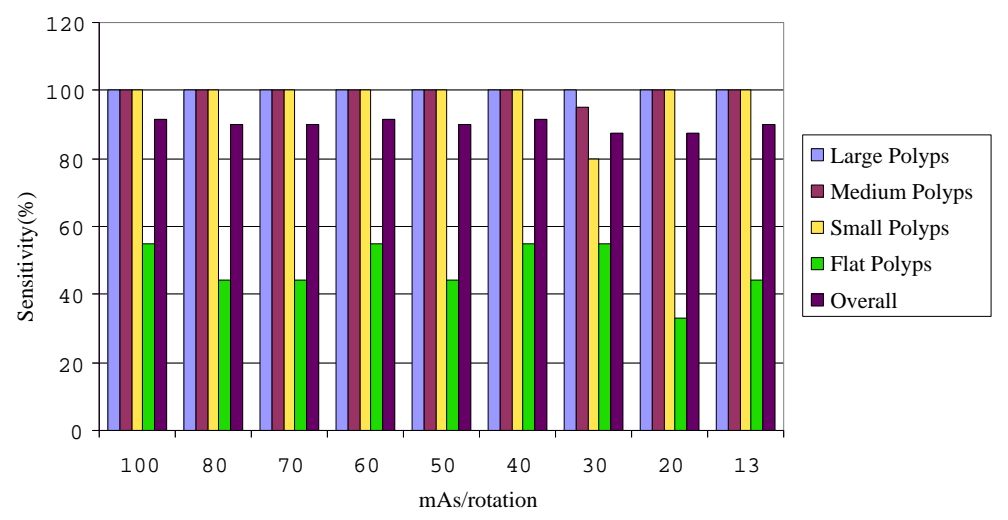

Fig. 7. Sensitivity of the polyp detection algorithm when applied to Protocol-3 CT data.

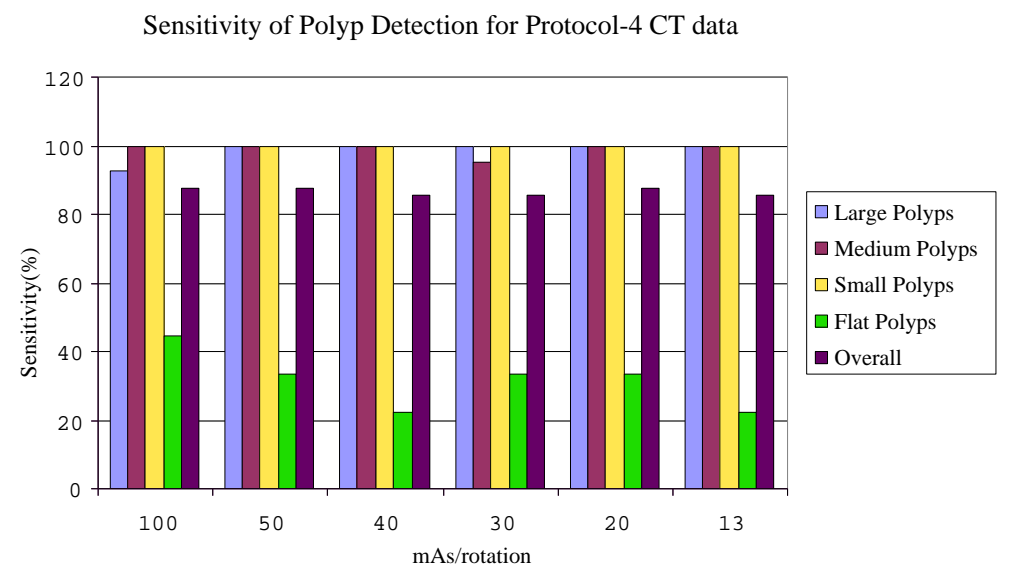

Fig. 8. Sensitivity of the polyp detection algorithm when applied to Protocol-4 CT data.

similar in all directions (voxel width and height: $0.7 \mathrm{~mm}$, voxel depth: $0.8 \mathrm{~mm}$ ). The experimental results indicate that the performance of the $C A D-C T C$ algorithm is virtually unchanged when it is applied to CT data acquired using the Protocols 1,3, 4 and 7. The results obtained when the algorithm has been applied to data acquired using the Protocol 5 were worse than those obtained when the algorithm was applied to CT data obtained using other protocols. 


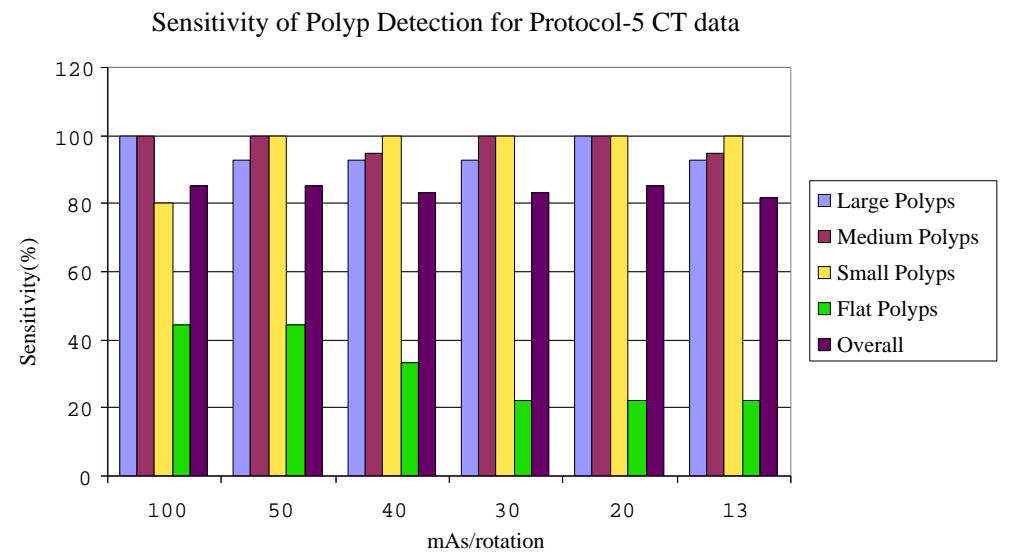

Fig. 9. Sensitivity of the polyp detection algorithm when applied to Protocol-5 CT data.

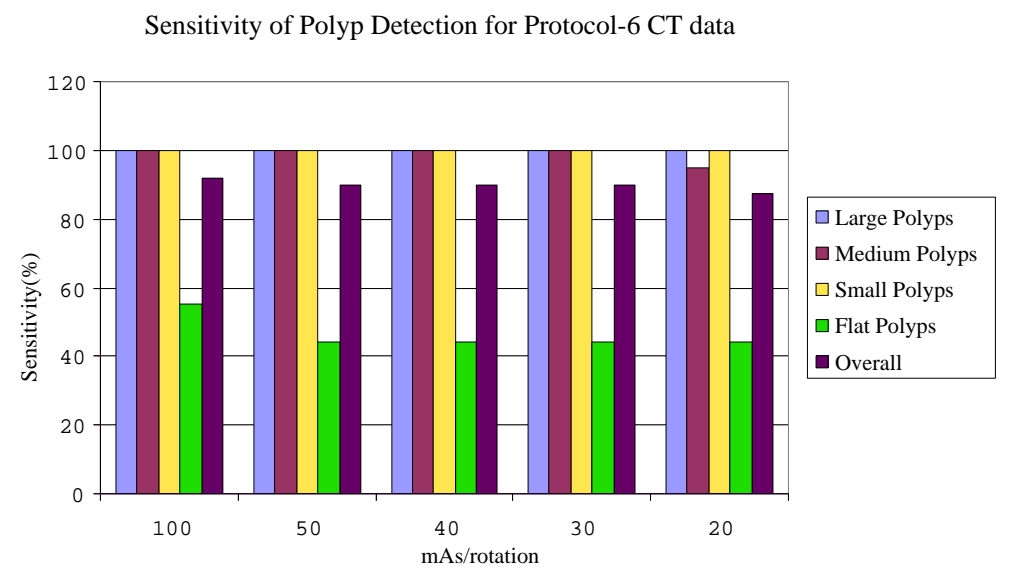

Fig. 10. Sensitivity of the polyp detection algorithm when applied to Protocol-6 CT data.

This has been generated by the fact that data interpolation has a smoothing effect on the 3D morphology of the colon wall and another important factor is that we have trained the classifier only with interpolated data.

Protocol 7 uses the collimation $0.75 \times 16 \mathrm{~mm}$ that allows us to scan the phantom at a slice thickness of $1 \mathrm{~mm}$ with a reconstruction interval of $0.7 \mathrm{~mm}$. This protocol was used to scan the phantom to create near isometric voxels and 


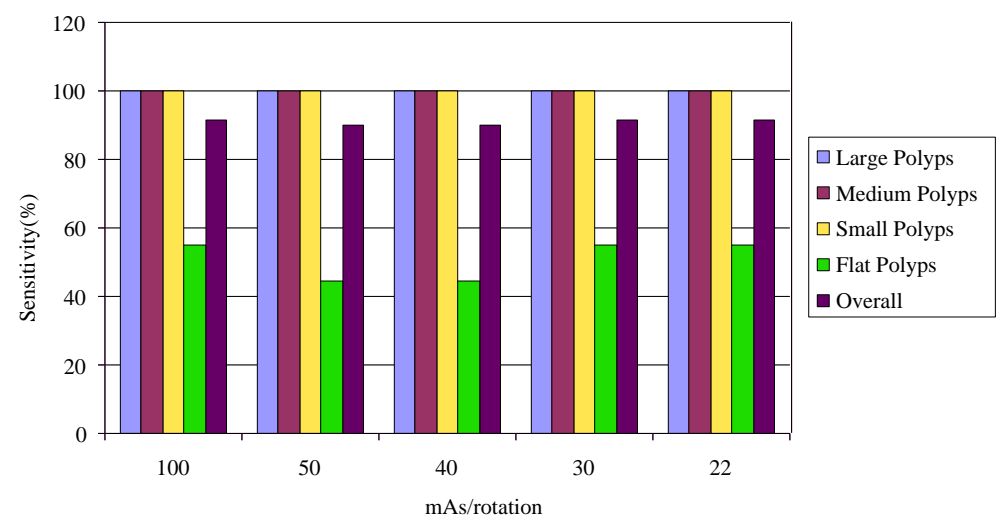

Fig. 11. Sensitivity of the polyp detection algorithm when applied to Protocol-7 CT data.

to evaluate the influence of the lower collimation on the overall performance of the $C A D-C T C$ system. Our automatic $C A D-C T C$ algorithm shows $100 \%$ sensitivity for polyps $\geq 10 \mathrm{~mm},[5-10) \mathrm{mm}$ and $<5 \mathrm{~mm}$ for all doses ranging from 22 to $100 \mathrm{mAs} /$ rotation. As indicated in Figure 11 our $C A D-C T C$ system shows higher sensitivity when applied to $30 \mathrm{mAs} /$ rotation CT data acquired using the Protocol $7(100 \%)$ than in cases when the phantom was scanned at the same radiation dose using the Protocols 1 to 5 . It is useful to note that the small increase in sensitivity noticed when the phantom was scanned using the Protocol 7 is obtained at the expense of a higher rate of false positives (generated by the uneven surface of the phantom) and higher radiation dose.

The field of view was set to $360 \mathrm{~mm}$ for Protocol 2 and to $325 \mathrm{~mm}$ for other protocols. The experimental data indicates that the field of view does not have a significant impact on the performance of the automated polyp detection algorithm.

Another parameter of interest is the table speed. To evaluate the influence of 
this parameter on the overall polyp detection results, we set this parameter to $20 \mathrm{~mm} /$ rotation for Protocol 6 and $30 \mathrm{~mm} /$ rotation for Protocols 1 to 5 and 7 . At $30 \mathrm{~mm} /$ rotation and $20 \mathrm{~mm} /$ rotation table speeds the effective dose is $2.7 \mathrm{mSv}$ at $100 \mathrm{mAs} /$ rotation for Protocols 1 to 6 . This parameter has a negligible effect on the radiation dose since the Siemens scanner used in our experiments utilises the "effective tube current" model where a variation in the scan time (the lower the scan time the higher the table speed) implies a concomitant variation in the tube current. For Siemens Somatom 16 slice CT scanner the lowest $\mathrm{mAs} /$ rotation that can be set at $20 \mathrm{~mm} /$ rotation table speed is $20 \mathrm{mAs} /$ rotation whereas for $30 \mathrm{~mm} /$ rotation table speed the lowest $\mathrm{mAs} /$ rotation is 13 . We have varied this parameter to evaluate only the effect of the motion artefacts and the experimental results indicate that the table speed has a marginal effect on the overall performance of our $C A D-C T C$ system. Small benefits have been observed when the algorithm has been applied to the detection of small (not clinically significant) and flat polyps.

\subsection{Level of noise and the radiation dose}

In this element of the study we aim to evaluate the correlation between the image noise and the radiation dose. In this regard we have selected five circular regions of interest (ROIs) with a radius of 20 voxels that are evaluated for 3 consecutive slices (see Figure 12). Since the data is homogenous (the phantom is filled with water) the level of noise can be accurately sampled by calculating the standard deviation (SD) of the voxel distribution within the circular region of interest.

For CT data scanned using the Protocols 1 and 3, the SD increased with a fac- 


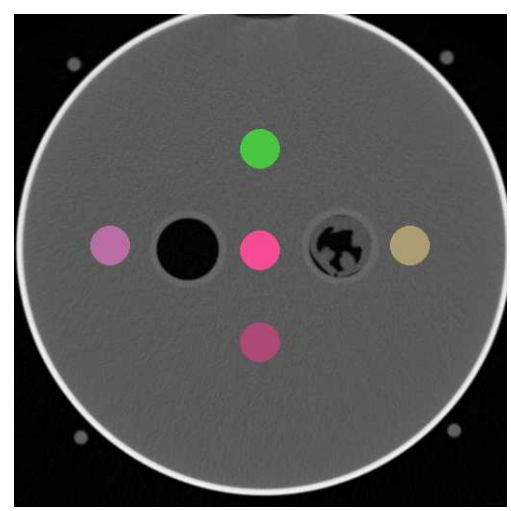

Fig. 12. Five regions of interests located on the phantom to evaluate the noise level.

tor of $2.67(\mathrm{SD}=26.59$ for $100 \mathrm{mAs} /$ rotation and $\mathrm{SD}=70.95$ for $13 \mathrm{mAs} /$ rotation $)$ when the scan was performed at $13 \mathrm{mAs} /$ rotation when compared to the case when the phantom was scanned with $100 \mathrm{mAs} /$ rotation radiation dose. The relation between the noise level and the radiation dose is almost linear and this is illustrated in Figure 13. In Figure 13 is noticed a small decay in the plot for Protocol 1 that may be caused by the smoothing effect induced by the data interpolation procedure.

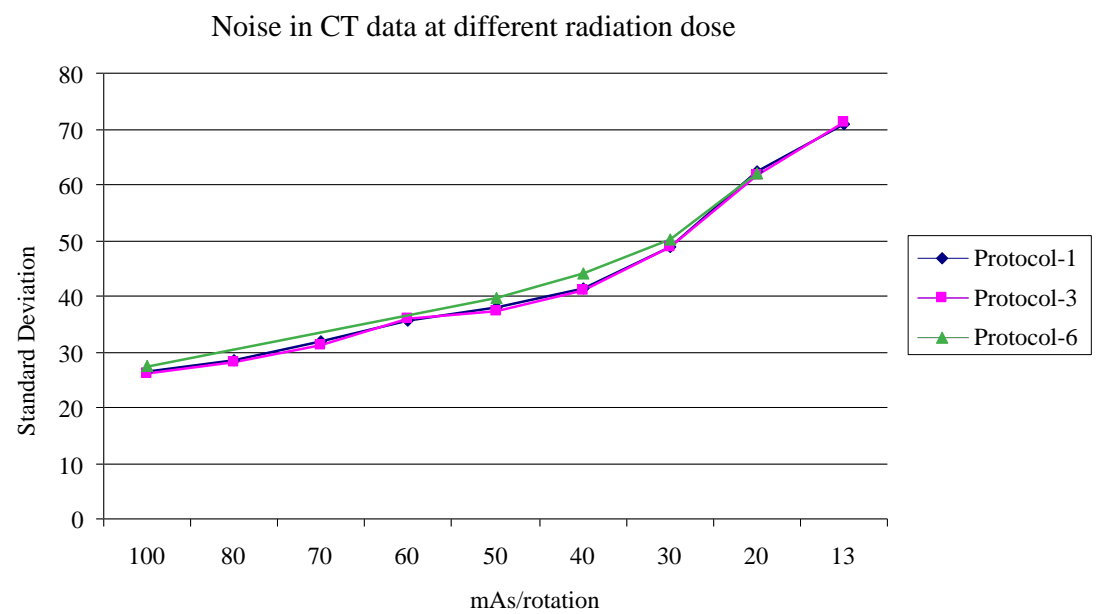

Fig. 13. The relationship between noise level and the radiation dose. 


\section{Conclusions}

The experimental data presented in this paper is obtained by scanning the synthetic phantom described in Section 2.1. Although the phantom was designed to emulate as closely as possible the real clinical conditions it is worth noting that the synthetic data is not affected by factors such as motion artefacts (caused by breathing) or the presence of residual material such as fluid and stool that are currently experienced when analysing real patient data. One of the main aims of this investigation was the development of a study environment that allows us to determine the influence of the scanning parameters on the performance of the polyp detection algorithm. Currently, the performance of the existing $C A D-C T C$ systems is evaluated on real patient data that is supplied by different research organizations that are not available for computer vision community. Therefore the absence of standard test data makes the performance evaluation of these systems restricted to the scenario they were tested. Thus, another important merit of this investigation is the generation of ground truth synthetic data that can be used to test all developed systems in the same conditions. For comparison purposes we have made the phantom data available on request from the following web page: http://www.eeng.dcu.ie/ whelanp/cadctc. Typical size of a CT dataset is in the range (70-125MB). It is useful to note that recently the Walter Reed Army Medical Center (WRAMC) database has been made available to the research community which will help the evaluation of the developed $C A D-C T C$ systems but the main advantage of using synthetic data is the generation of unambiguous ground truth data (requires no validation by radiologists) that can be used especially in the development phase of the $C A D-C T C$ systems. 
Our $C A D-C T C$ system indicates that automated polyp detection is feasible even at radiation doses as low as $13 \mathrm{mAs} /$ rotation. The sensitivity rate in polyp detection achieved by our $C A D-C T C$ system is always higher than $90 \%$ for polyps larger than $5 \mathrm{~mm}$ and the overall sensitivity for all types of polyps is higher than $80 \%$. The sensitivity rate would be even higher as our method has not been trained for the detection of flat polyps. For this type of polyps the achieved sensitivity is in the range $22 \%$ to $55 \%$. In our experiments one polyp (see Figure 4a) has been placed closed to the outer plastic plates of the phantom and at low radiation doses the image noise joined the surface of the polyp with the surface generated by the plastic plate and the classifier assigned this surface to be part of a fold. It is worth mentioning that this situation will not appear in clinical studies.

The main merit of this paper is the development of a realistic phantom that closely simulates the situations encountered in real clinical studies. Thus, we placed the main emphasis on evaluating the influence of the scanning parameters on the performance of the automated polyp detection. From these parameters we focused our attention on the radiation dose as the main concern regarding $\mathrm{CT}$ examinations is the exposure of the patients to ionizing radiation. Recent studies demonstrated that $\mathrm{CT}$ which accounts for $4 \%$ of the medical radiographic examinations contributes $35-40 \%$ of the cumulated radiation dose received by the patients [64]. Our study reveals that the reduction of $\mathrm{mAs} /$ rotation from 100 to $13(1.5 \times 16 \mathrm{~mm}$ collimation $)$ reduced the effective dose from $2.7 \mathrm{mSv}$ to $0.35 \mathrm{mSv}$ as it is illustrated in Figure 14 . In our experiments we have also scanned the phantom using a reduced collimation $(0.75 \times 16 \mathrm{~mm})$ but the experimental data indicates that the small increase in polyp detection sensitivity achieved by our $C A D-C T C$ system does not 
justify the increased radiation dose that would be received by patients (there will be an $11 \%$ increase of the effective dose as illustrated in Figure 14). In addition it is worth noting that the volume of $\mathrm{CT}$ data acquired at a reduced collimation is significantly larger than the volume of CT data generated at $1.5 \times 16 \mathrm{~mm}$ collimation and this will be a deterring factor when the data is evaluated manually by radiologists. We conclude that a reduced collimation is not justified since the increase in sensitivity is marginal and for clinical purposes a $1.5 \times 16 \mathrm{~mm}$ collimation is deemed appropriate to detect the clinically significant colorectal polyps (see the results reported in Figure 5 to 10 $(1.5 \times 16 \mathrm{~mm}$ colimation $)$ and Figure $11(0.75 \times 16 \mathrm{~mm}$ collimation $))$.

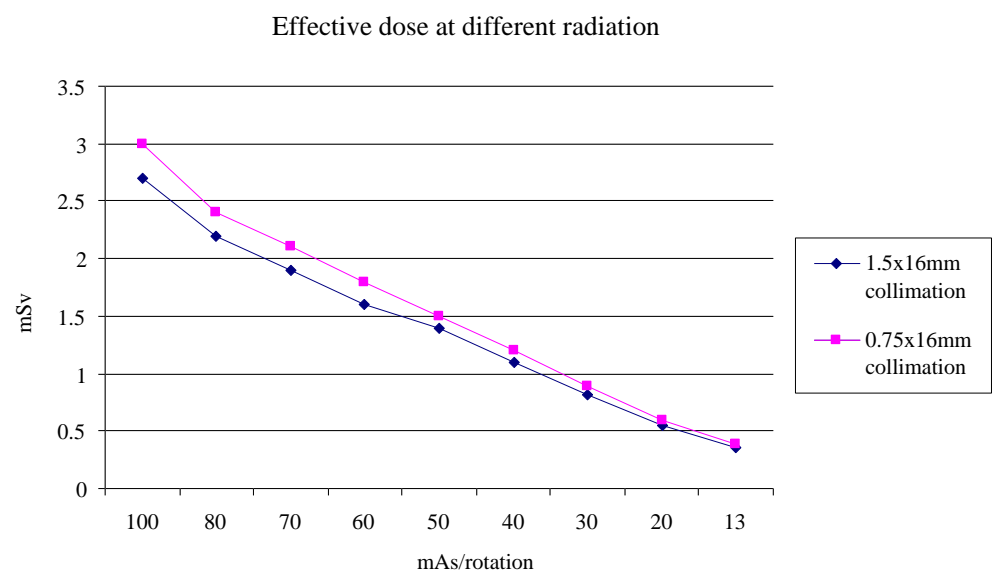

Fig. 14. Radiation dose received by the patient at different $\mathrm{mAs} /$ rotation using the ImPACT dosimetry tool [65]

Also another important issue we tried to address in this paper is the relationship between the radiation dose and the impact on the performance of the $C A D-C T C$ polyp detection algorithm. In this regard, our studies indicated that the level of image noise when the phantom was scanned with $13 \mathrm{mAs} /$ rotation was higher with a factor of 2.67 than in the case when the phantom was scanned with $100 \mathrm{mAs} /$ rotation radiation dose. Although the 
level of noise significantly increased at low radiation dose the effect on the performance in polyp detection is minimal. The experimental data presented in Figures 5 to 11 indicates that the sensitivity in polyp detection for polyps larger than $5 \mathrm{~mm}$ is always above $95 \%$. We notice a small increase in false positives at $13 \mathrm{mAs} /$ rotation but the effect on true positive detection rate is not noticeable. The impact of the field of view and the reconstruction interval was negligible and it was virtually eliminated by the smoothing effect of the data interpolation that is applied to make the dataset isometric. We conclude that low dose radiation (as low as $13 \mathrm{mAs} /$ rotation) is feasible to be used in standard CTC clinical examinations.

\section{Acknowledgements}

We would like to acknowledge the contributions of our clinical partners in this project: Dr. Helen Fenlon (Department of Radiology) and Dr. Padraic MacMathuna (Gastrointestinal Unit) of the Mater Misericordiae Hospital, Dublin. We would also like to acknowledge our colleague Robert Sadleir for the development of the DICOM decoder software. This work was supported under an Investigator Programme Grant (02/IN1/1056) by Science Foundation Ireland (SFI). Finally, we would like to thank the anonymous reviewers for their helpful feedback.

\section{References}

[1] Parker S., Tong T., Bolden S., Wingo P., Cancer statistics 1997, CA Cancer Journal for Clinicians, 1997, 47, 5-27. 
[2] NCRI, 2000: Cancer in Ireland, Incidence and Mortality, Healy \& Associates, 1997.

[3] Cancer Research UK, Bowel Cancer Factsheet April, 2003.

[4] Ransoho D.F., Sandler R.S., Screening for Colorectal Cancer, The New England Journal of Medicine, January 3, 2002, 346(1).

[5] American Cancer Society, Cancer Facts and Figures, American Cancer Society, 1999.

[6] National Cancer Institute, Working guidelines for early cancer detection: Rationale and supporting evidence to decrease mortality, Bethesda: National Cancer Institute, 1987.

[7] Robert A., Cokkinides S. V., Eyre H.J., American Cancer Society Guidelines or the Early Detection of Cancer, A Cancer Journal for Clinicians, 2003, 53, $27-43$.

[8] Schrock T.R., Colonoscopy versus barium enema in the diagnosis of colorectal cancer and polyps, GastroIntestinal Endoscopy Clinics of North America, 1993, $3,585-610$.

[9] Winawer J.S., Stewart E.T., Zauber A.G., Bond J.H., A Comparison of Colonoscopy and Double-Contrast Barium Enema for Surveillance After Polypectomy, The New England Journal of Medicine, 2000, 342, 1766-1772.

[10] Sato M., Lakare S., Wan M., Kaufman A., Liang Z., Wax M., An Automatic colon segmentation for 3D virtual colonoscopy, IEICE Transactions Information and Systems, January, 2001, Vol. E84-D, No. 1, 201-208.

[11] Vining D.J., Gelfand D.W., Bechtold R.E., Scharling E.S., Grishaw E.K., Shifrin R.Y., Technical feasibility of colon imaging with helical CT and virtual reality, American Journal of Roentgenology, 1994, 162, 104. 
[12] Johnson C.D., Hara A.K., Reed J.E., Virtual endoscopy: what's in a name?, American Journal of Roentgenology, 1998, 171, 1201-2.

[13] Lichan H., Arie K., Chih W. Yi, Ajay V., Mark W., L. Zhengrong, 3D Virtual Colonoscopy, In Proceeding of Biomedical Visualization, M. Loew and N. Gershon, Eds., 1995, Atlanta, 26-33.

[14] Hara A.K., Johnson C.D., Reed J.E., Ahlquist D.A., Nelson H., Ehman R.L., Detection of colorectal polyps by CT colonography: Feasibility of a novel technique, Gastroenterology, 1996, 100, 284-290.

[15] Hong L., Muraki S., Kaufman A., Bartz D., He T., Virtual voyage: Interactive navigation in the human colon, Proceeding of ACM SIGGRAPH, August 1997, Los Angeles, 27-34.

[16] Wan M., Tang Q., Kaufman A., Liang Z., Wax M., Volume rendering based interactive navigation within the human colon, Proceeding of IEEE Visualization, San Francisco, 1999, pp. 397-400.

[17] Sezille N., Sadleir R.J.T, Whelan P.F., Fast extraction of planes normal to the centreline from CT colonography datasets, VIE 2003 - IEE Visual Information Engineering Conference, Guildford, UK, 2003.

[18] Sadleir R.J.T., Whelan P.F., Fast Colon Centreline Calculation using Optimised 3D Topological Thinning, Computerized Medical Imaging and Graphics, June 2005, 29(4), 251-258.

[19] Bartroli A.V., Wegenkittl R., Konig A., Groller E., Nonlinear Virtual Colon Unfolding, 12th IEEE Visualization 2001 (VIS'01).

[20] Vining D.J., Hunt G.W., Ahn D.K., Stelts D.R., Helmer P.F., Computer assisted detection of colon polyps and masses, Radiology, 2001, 219, 51-59.

[21] Summers R.M., Johnson C.D., Pusanik L.M., Malley J.D., Youssef A.M., Reed 
J.E., Automated polyp detection at CT colonography: Feasibility assessment in a human population, Radiology, 2001, 219, 51-59.

[22] Summers R.M., Beaulieu C.F., Pusanik L. M., Malley J.D., Jeffrey R.B., Glazer D.I., Napel S., Automated Polyp Detector for CT Colonography: Feasibility Study, Radiology, 2000, 216, 284-290.

[23] Yoshida H., Masutani Y., MacEneaney P., Rubin D.T., Dachman A.H., Computerized detection of colonic polyps at $\mathrm{CT}$ colonography on the basis of volumetric features: Pilot study, Radiology, 2002, vol. 222, pp. 327-336.

[24] Yoshida H., Nappi J., Three-Dimensional computer-aided diagnosis scheme for detection of colonic polyps, IEEE Transactions on Medical Imaging, 2001, 20(12), 1261-1274.

[25] Paik D.S., Beaulieu C.F., Rey R.B.J., Computer aided detection of polyps in CT colonography: Method and free-response ROC evaluation of performance, Radiology, 2000, 217(P), 370.

[26] Kiss G., Cleynenbreugel J., Thomeer M., Suetens P., Marchal G., Computer aided diagnosis for virtual colonography, Medical Image Computing and Computer-Assisted Intervention, 2001, 621-628.

[27] Kiss G., Cleynenbreugel J., Thomeer M., Suetens P., Marchal G., Computer aided detection of colonic polyps via geometric feature classication, Vision, Modeling, and Visualization, 2002, 27-34.

[28] Kiss G., Cleynenbreugel J., Suetens P., Marchal G., Computer aided diagnosis for CT colonography via slope density functions, Medical Image Computing and Computer-Assisted Intervention 2003, 746-753.

[29] Paik D.S., Beaulieu C.F., Rubin G.D., Acar B., Jeffrey R.B., Jr., Yee J., Dey J., Napel S., Surface normal overlap: a computer-aided detection algorithm with 
application to colonic polyps and lung nodules in helical CT, IEEE Transactions on Medical Imaging, 2004, 23(6), 661-75.

[30] Kiraly A.P., Laks S., Macari M., Geiger B., Bogoni L., Novak C.L., A fast method for colon polyp detection in high-resolution CT data, International Congress Series, 2004, 1268, 983-988.

[31] Acar B., Napel S., Paik D., Gokturk S.B., Tomasi C., Beaulieu C.F., Using optical flow fields for polyp detection in virtual colonoscopy, Medical Image Computing and Computer-Assisted Intervention, Utrecht, Holland, 2001.

[32] Wang Z., Li L., Anderson J., Harrington D., Liang Z., Colonic polyp characterization and detection based on both morphological and texture features, International Congress Series, 2004, 1268, 1004-1009.

[33] Jerebko A.K., Malley J.D., Franaszek M., Summers R.M., Multi neural network classification scheme for detection of colonic polyps in CT colonography data sets, Academic Radiology, 2003, 10(2), 154-160.

[34] Chowdhury T.A., Ghita O., Whelan P.F., A statistical approach for robust polyp detection in CT colonography, 27th Annual International Conference of the IEEE Engineering in Medicine and Biology Society, 1-4 September 2005, Shanghai, China.

[35] Barnes E., Colon CAD: VC's extra eyes face new challenges, AuntMinnie.com, August 5, 2005.

[36] Yee J., Akerkar G.A., Hung R.K., Steinauer-Gebauer A.M., Wall S.D., McQuaid K.R., Colorectal Neoplasia: Performance Characteristics of CT Colonography for Detection in 300 Patients, Radiology, 2001, 219, 685.

[37] Fletcher J.G., Johnson C.D., Krueger W.R., Ahlquist D.A., Nelson H., Ilstrup D., Harmsen W.S., Corcoran K.E., Contrast-Enhanced CT Colonography in Recurrent Colorectal Carcinoma: Feasibility of Simultaneous Evaluation 
for Metastatic Disease, Local Recurrence, and Metachronous Neoplasia in Colorectal Carcinoma, American Journal Roentgenol, 178, 283-290, Feb 2002.

[38] Hoon J., Rolnick J.A., Haker S., Barish M.A., Multislice CT colonography: Current status and limitations, European Journal of Radiology, August 2003, $47(2), 123-134$.

[39] Sosna J., Morrin M. M., Kruskal J. B., Farrell R. J., Nasser I., Raptopoulos V., Colorectal Neoplasms: Role of Intravenous Contrast-enhanced CT Colonography, Radiology, 2003, 228(1), 152-156, July 1.

[40] Filippone A., Ambrosini R., Fuschi M., Marinelli T., Genovesi D., Bonomo L., Preoperative T and N Staging of Colorectal Cancer: Accuracy of Contrastenhanced Multi-Detector Row CT Colonography-Initial Experience, Radiology, April 1, 2004 231(1), 83-90.

[41] Gelder R.E.V., Venema H.W., Florie J., Yung C., Serlie I.W.O., Schutter M.P., Rijn J.C.V., Vos F.M., Glas A.S., Bossuyt P.M.M., Bartelsman J.F.W., Lameris J.S., Stoker J., CT Colonography: Feasibility of Substantial Dose ReductionComparison of Medium to Very Low Doses in Identical Patients, Radiology, 2004, 232, 611-620.

[42] Brenner D.J., Elliston C.D., Hall E.J., Estimated risks of radiation induced fatal cancer from pediatric CT, American Journal of Roentgenology, February 2001, 176, 289-296.

[43] Cohen B.L., Cancer risk from low-level radiation, American Journal of Roentgenology, 2002, 179, 1137-1143.

[44] Iannaccone R., Laghi A., Catalano C., Brink J.A., Mangiapane F., Trenna S., Piacentini F., Passariello R., Detection of Colorectal Lesions: Lower-Dose Multi-Detector Row Helical CT Colonography Compared with Conventional Colonoscopy, Radiology, 2003, 229, 775-781. 
[45] Capunay C.M., Carrascosa P.M., Bou-Khair A., Castagnino N., Ninomiya I., Carrascosa J.M., Low radiation dose multislice CT colonography in children: Experience after 100 studies, European Journal of Radiology, In Press, Available online 5 July 2005.

[46] Vogt C., Cohnen M., Beck A., Dahl S.V., Aurich V., Modder U., Haussinger D., Detection of colorectal polyps by multislice CT colonography with ultra-low-dose technique: Comparison with high-resolution videocolonoscopy, Gastrointestinal Endoscopy, August 2004, 60(2), 201-209.

[47] Beaulieu C.F., Napel S., Daniel B.L., Ch'en I.Y., Rubin G.D., Johnstone I.M., Jeffrey, Jr.B., Detection of colonic Polyps in a phantom model: Implications for virtual colonoscopy data acquisition, Journal of Computer Assisted Tomography, 1998, 22(4), 656-663.

[48] Dachman A.H., Lieberman J., Osnis R.B., Chen S.Y., Hoffmann K.R., Chen C.T., Newmark G.M., McGill J., Small simulated polyps in pig colon: Sensitivity of CT virtual colography, Radiology, 1997, 203, 427-430.

[49] Taylor S.A., Halligan S., Bartram C.I., Morgan P.R., Talbot I.C., Fry N., Saunders B.P., Khosraviani K., Atkin W., Multi-detector row CT colonography: Effect of collimation, pitch, and orientation on polyp detection in Human Colectomy Specimen, Radiology, 2003, 229, 109-118.

[50] Springer P., Stohr B., Giacomuzzi S.M., Bodner G., Klingler A., Jaschke W., Nedden D. zur, Virtual computed tomography colonoscopy: Artifacts, image quality and radiation dose load in a cadaver study, European Radiology 2000, 10(1), 183-7.

[51] Whiting B.R., McFarland E.G., Brink J.A., Influence of Image Acquisition Parameters on CT Artifacts and polyp detection in spiral CT colonography: In Vitro Evalution, Radiolgy, 2002, 217, 165-172. 
[52] Power N.P., Pryor M.D., Martin A., Horrocks J., McLean A.M., Reznek R.H., Optimization of scanning parameters for CT colonography, The British Journal of Radiology, 2002, 75, 401-408.

[53] Wessling J., Fischbach R., Meier N., Allkemper T., Klusmeier J., Ludwig K., Heindel W., CT colongraphy: Protocol optimization with Muti-detector row CT-study in an Anthropomorphic Colon Phantom, Radiology, 2003, 228, 753759.

[54] Laghi A., Iannaccone R., Mangiapane F., Piacentini F., Iori S., Passariello R., Experimental colonic phantom for the evaluation of the optimal scanning technique for CT colonography using a multi-detector spiral CT equipment, European Radiology 2003, 13(3), 459-466.

[55] Embleton K.V., Nicholson D.A., Hufton A.P., Jackson A., Optimization of scanning parameters for multi-slice CT colonography: experiments with synthetic and animal phantoms, Clinical radiology December 2003, 58(12), 95563.

[56] Ozgun A., Rollven E., Blomqvist L., Bremmer S., Odh R., Fransson A., Polyp Detection with MDCT: A Phantom-based Evaluation of the Impact of dose and spiral Resolution, April 2005, American Journal of Roentology, 184.

[57] Ling S.H., Summers R.M., Loew M.H., McCollough C.H., Johnson C.D., Computer-aided detection of polyps in a colon phantom: Effects of scan orientation, polyps size, collimation and dose, Journal of Computer Assisted Tomography, 2002, 26(6), 1013-1018.

[58] Sundaram P., C.F. Beaulieu, Paik D.S., Schraedley-Desmond P., Napel S., CT colonography: Does improved z resolution help computer-aided polyp detection?, Medical Physics, Oct. 2003, 30(10), 2663-74.

[59] NHS Purchasing and Supply Agency, Report 05071, Siemens Somatom 
Sensation open CT scanner technical evaluation, December 2005.

[60] Chowdhury T.A., Whelan P.F., Ghita Ovidiu, A Method for Automatic Segmentation of Collapsed Colons at CT Colonography, 2nd Indian International Conference on Artificial Intelligence, 2005, December 20-22.

[61] Zucker S.W., Hummel R.A., A Three-Dimensional edge operator, IEEE Transactions on Pattern Analysis and Machine Intelligence, 1981, 3(3), 324331.

[62] Ghita O., Whelan P.F., A bin picking system based on depth from focus, Machine Vision and Application, 2003, 13, 234-244.

[63] Park S.H., Ha H.K., Kim A.Y., Kim K.W., Lee M.G., Kim P.N., Shin Y.M., Byeon J.S., Yang S.K., Kim J.H., Min Y.I., Flat polyps of the colon: Detection with 16-MDCT colonographypreliminary results, American Journal of Roentology, 2006, 186, 1611-1617.

[64] Nagel H.D., Radiation Exposure in computed tomography, European Coordinate Committee of the Radiological and Electromedical Industries, Frankfurt, Germany, COCIR, 2000.

[65] ImPACT CT Patient Dosimetry $\quad$ Calculator. www.impactscan.org/ctdosimetry.htm. 